\title{
A case report of Baboon Syndrome due to azithromycin
}

\section{Munise Daye ${ }^{1}$, Koray Durmaz', Pembe Oltulu²}

${ }^{1}$ Department of Dermatology, Necmettin Erbakan University Meram Faculty of Medicine, Konya, Turkey, ${ }^{2}$ Department of Pathology, Necmettin Erbakan University Meram Faculty of Medicine, Konya, Turkey

Corresponding author: Dr. Munise Daye, E-mail: dr_munise@yahoo.com

\begin{abstract}
Baboon syndrome is a type IV delayed hypersensitivity reaction and characterized by asymptomatic erythematous maculopapular rash due to drugs. The rash typically involves flexural areas, gluteal and perineal regions. This eruption, may occur within hours to days of exposure to suspecting drug and resolves rapidly by discontinuation of the triggering drug. We present a 76-years-old male patient who admitted to our clinic with symettrical, erythematous eruption and was diagnosed Baboon syndrome by clinical and histopathological findings. The eruption disappeared in 1 week after appropriate treatment via intravenous methyl prednisolone, oral antihistamines and topical steroids.
\end{abstract}

Key words: Dermatitis; Drug eruption; Azithromycin

\section{INTRODUCTION}

Symmetrical drug related intertriginous and flexural exanthema (SDRIFE) or Baboon syndrome (BS) is a form of systemic contact dermatitis represents a delayed hypersensitivity reaction and desribed with exanthematous involvement of gluteal and intertriginous areas after ingestion or systemic absorption of a contact allergen in a sensitized individual [1-3]. We report a case of a 76-year-old male patient with BS after systemic use of azithromycin.

\section{CASE REPORT}

A-76-year-old male patient was submitted to our clinic with a 10 days history because of erythematous eruption on his neck, dorsum of hands, forearms and penis and scrotal area. His past medical history was significant for a recently diagnosed upper respiratory tract infection, pharyngitis for whisch he was prescribed azithromycin $500 \mathrm{mg}$ tablets once a day for 3 days about 10 days prior. On the third day of initiating treatment with azithromycin the lesions started on his neck and dorsum of hands and spread to his inner thigs, antecubital regions of forearms, penis and scrotal areas in 3 days (Fig. 1). According to his previous medical record, he had not been prescribed topical and/or oral azithromycin before. There was no personal and/or family history of atopic diatheses or any other drug hypersensitivity. At first examination there was mild dyspnea due to asthma, but no fever or another systemic symptoms. Complete blood count, sedimentation, CRP, routine biochemistry including thyroid, hepatic and renal function tests, urinalyses were normal. Histopathologic examination of the biopsy from the affected site of the left forearm showed pustule formation at the subcorneum and variable superficial perivascular infiltrate of eosinophils in the dermis (Fig. 2). After clinicopathologic correlation, the patient was diagnosed BS (SDRIFE). He was treated with $40 \mathrm{mg}$ methyl prednisolone for 3 days and then $20 \mathrm{mg}$ methyl prednisolone for 5 days intravenous infusion. Mild topical steroids and systemic antihistamines was also added to treatment. The eruption appeared to have cleared with desquamation in 1 week and patient's subjective symptoms like burning and pruritus was resolved (Fig. 3).

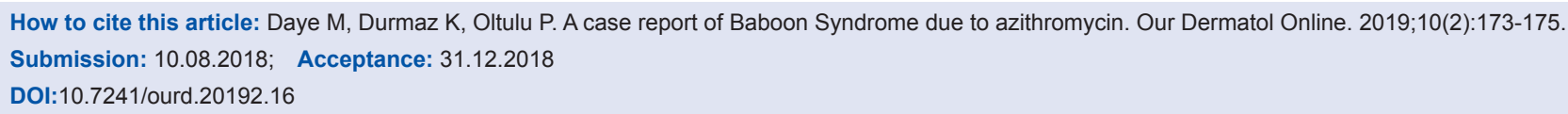




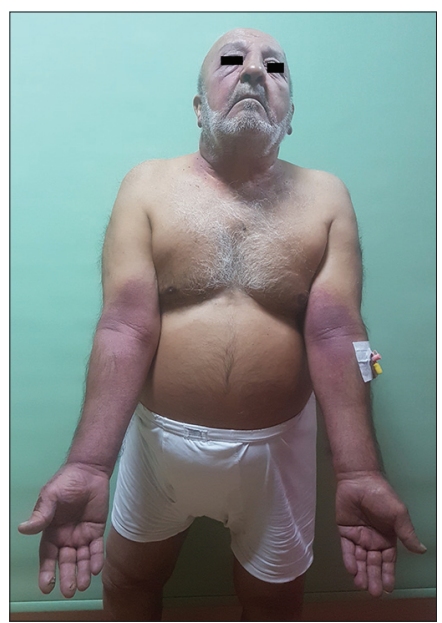

Figure 1: Symmetrical eryhthematous rash of the bilateral flexural regions and neck involvement.

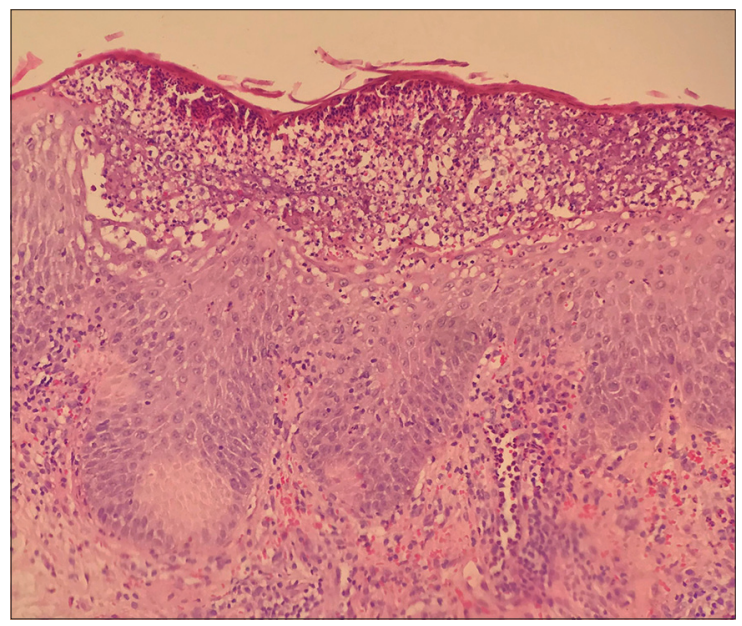

Figure 2: Pustule formation at the subcorneum and superficial perivascular infiltrate of eosinophils in the dermis.

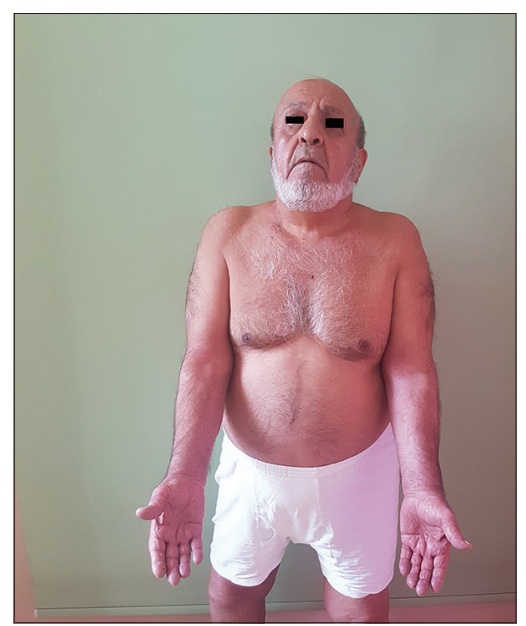

Figure 3: The eruption dramatically resolved after the treatment.

Prior to the study, patient gave written consent to the examination and biopsy after having been informed about the procedure.

\section{DISCUSSION}

BS term was introduced in 1984 by Andersen et al. as a peculiar form of systemic contact dermatitis characterized by symmetric and diffuse erythematous maculopapular eruption on the buttocks and flexural areas after re-exposure of a contact allergen. Andersen and his coauthors presented three patients who had developed generalized erythematous maculopapular rash located in glutea and intertriginous areas, provoked by to mercury, nickel and ampicillin. They named the eruption as BS cause of lesional presentation of the patient's buttocks like to the red appeareance of a baboon [4]. As we can see from reported cases in the literature BS mostly presented 18 months of age to 84 years old patients, it can be seen at any age and both sexes. SDRIFE (symmetrical drug-related intertriginous and flexural exanthema) term has also been used as acronym BS in many literatures, proposed to describe such cases of BS occuring after systemic exposure of drugs and contact allergens. Although the precise pathogenetic mechanisms are still unknown for this reaction, it has been suspected to develop as a result of a T-cell mediated type IV hypersensitivity reaction. Diagnostic criteria for SDRIFE and clinical presentations are (I) Exposure to a systemically administered drug for the first time or repeated doses (contact allergens are excluded); (II) sharply bordered erythema of the gluteal/perineal areas and/or, V-shaped erythema located on the inguinal/perigenital areas; (III) in addition, involvement of at least one different intertriginous/flexural region; (IV) symmetry and (V) Absence of any systemic symptoms and signs such as pyrexia or eosionophili. SDRIFE, mostly observed after to beta-lactam antibiotics such as penicillines or cephalosporine. The diagnosis of this eruption is generally based on the clinical presentation and medical history of patients. Suspected sensitizing agents cannot be found in most of cases, patch tests or prick tests are negative in $1 / 3-1 / 2$ of patients and predictive values of the tests depends on the clinical features of the adverse reaction and on the particular drug tested. The histopathology of SDRIFE typically shows an superficial perivascular infiltrate including neutrophils and eosinophils, on the other hand subcorneal pustules, vacuolar changes and hydropic degeneration in the basal cell layer were also described $[3,5,6]$. The latency period generally varies from hours to a few days after exposure to the suspected agent. In our case, this duration was three days and the patient's lesions started on his neck and dorsum of hands and spread to inner 
face of thigs, antecubital regions, scrotal and pubic areas in a few days. He has not any systemic symptoms and eosionophili too. In differential diagnosis, fixed drug eruption (FDE) excluded absence of acral, genital or mucosal round oval plaques and bullous lesions. We did not consider other systemic drug eruptions like acute generalized exanthematous pustulosis (AGEP) or drug rash with eosinophilia (DRESS) absence of a widespread rash with accompanying systemic changes in this case. We diagnosed BS(SDRIFE), the patient's clinical presentation and histopathological findings such as pustule formation at the subcorneum and variable superficial perivascular infiltrate of eosinophils in the dermis.

\section{CONCLUSION}

The inciting agent causing BS (SDRIFE) in our case was azithromycin which is considered one of the safest antibiotic and widely prescribed for acute respiratory tract infection. Even if the major side effects of Azithromycin are mild to moderate in severity and related to the gastrointestinal symtoms and reversible hearing loss, it is usually well-tolerated by patients. Drug related rash in Azithromycin usage is observed about $6 \%$ of cases. Severe cutaneous reactions have been rarely reported in the literature [7].

This condition is a benign drup eruption that cleared within days after stopping of the offending agent [8]. To our knowledge, this is the first reported case of BS (SDRIFE) associated with azithromycin.

\section{Consent}

The examination of the patient was conducted according to the Declaration of Helsinki principles.

\section{REFERENCES}

1. Culav I, Ljubojevic S, Buzina DS. Baboon syndrome/SDRIFE due to sulfamethoxazole-trimethoprim. Int J Dermatol. 2013;52:1159-60.

2. Winnicki M, Shear NH. A systematic approach to systemic contact dermatitis and symmetric drug-related intertriginous and flexural exanthema (SDRIFE): a closer look at these conditions and an approach to intertriginous eruptions. Am J Clin Dermatol. 2011;12:171-80.

3. Häusermann P, Harr T, Bircher AJ. Baboon syndrome resulting from systemic drugs: is there strife between SDRIFE and allergic contact dermatitis syndrome?. Contact Derm. 2004;51:297-310.

4. Wolf R, Tüzün Y. Baboon syndrome and toxic erythema of chemotherapy: Fold (intertriginous) dermatoses. Clin Dermatol. 2015;33:462-5.

5. Hausermann P, Bircher AJ. SDRIFE - another acronym for a distinct cutaneous drug exanthema: do we really need it? Dermatology. 2007;214:1-2.

6. Lachapelle J-M. The spectrum of diseases for which patch testing is recommended. In: Lachapelle J-M, Maibach HI, eds. Patch Testing and Prick Testing: A Practical Guide. Official Publication of the ICDRG, $2^{\text {nd }}$ edn. Berlin: Springer-Verlag, 2009: 7-31.

7. Sriratanaviriyakul N, Nguyen LP, Henderson MC, Albertson TE. Drug reaction with eosinophilia and systemic symptoms syndrome (DRESS) syndrome associated with azithromycin presenting like septic shock: a case report. J Med Case Rep. 2014;8:332.

8. Dhingra B, Grover C. Baboon syndrome. Indian Pediatr. 2007;44:937.

Copyright by Munise Daye, et al. This is an open-access article distributed under the terms of the Creative Commons Attribution License, which permits unrestricted use, distribution, and reproduction in any medium, provided the original author and source are credited.

Source of Support: Nil, Conflict of Interest: None declared. 